University of Nebraska - Lincoln

DigitalCommons@University of Nebraska - Lincoln

Timothy J. Gay Publications

Research Papers in Physics and Astronomy

April 1981

\title{
Molecular effects in beam-foil collision-induced alignment of He I
}

Timothy J. Gay

University of Nebraska - Lincoln, tgay1@unl.edu

H. G. Berry

Argonne National Laboratory, Argonne, Illinois

R. DeSerio

Argonne National Laboratory, Argonne, Illinois

Follow this and additional works at: https://digitalcommons.unl.edu/physicsgay

Part of the Physics Commons

Gay, Timothy J. ; Berry, H. G.; and DeSerio, R. , "Molecular effects in beam-foil collision-induced alignment of He I" (1981). Timothy J. Gay Publications. 9.

https://digitalcommons.unl.edu/physicsgay/9

This Article is brought to you for free and open access by the Research Papers in Physics and Astronomy at DigitalCommons@University of Nebraska - Lincoln. It has been accepted for inclusion in Timothy J. Gay Publications by an authorized administrator of DigitalCommons@University of Nebraska - Lincoln. 
Phys. Rev. A 23, 1761 - 1774 (1981) [Issue 4 - April 1981]

\title{
Molecular effects in beam-foil collision-induced alignment of He I
}

\author{
T. J. Gay ${ }^{*} \dagger$, H. G. Berry, and R. DeSerio *
}

Physics Division, Argonne National Laboratory, Argonne, Illinois 60439

Received 28 July 1980

We have measured the alignment of beam-foil collision-excited states of He I produced by bombarding carbon foils of various $\left(1.3-110 \mu \mathrm{g} / \mathrm{cm}^{2}\right)$ areal densities with beams of $\mathrm{He}^{+}$and $\mathrm{HeH}^{+}$. In addition, we have measured the total light yield of several transitions in He I, He II, and $\mathrm{H}$ as a function of foil thickness using beams of $\mathrm{HeH}^{+}$ions. Experiments were done with He-foil exit energies of $125,500,550$, and $650 \mathrm{keV}$. He I alignment decreases in all cases for the thinnest foils when molecular projectiles are used. Total light intensities generally increase with thin foils (small proton-He emergent internuclear separation), but a few decrease or are independent of foil thickness. We are able to explain several features of the alignment and intensity data in terms of the formation of quasimolecular $\mathrm{HeH}^{+}$states at or near the foil surface. Alignment reduction results from incoherent Stark mixing of the He I excited states in the field of the close proton. A calculation of the rms emergent $\mathrm{H}-\mathrm{He}$ internuclear separation as a function of foil thickness and beam energy is presented.

(C)1981 The American Physical Society

URL: http://link.aps.org/abstract/PRA/v23/p1761

DOI: 10.1103/PhysRevA.23.1761 


\title{
Molecular effects in beam-foil collision-induced alignment of $\mathrm{He}$ I
}

\author{
T. J. Gay, ${ }^{* \dagger}$ H. G. Berry, and R. DeSerio* \\ Physics Division, Argonne National Laboratory, Argonne, Illinois 60439
}

(Received 28 July 1980)

\begin{abstract}
We have measured the alignment of beam-foil collision-excited states of He I produced by bombarding carbon foils of various $\left(1.3-110 \mu \mathrm{g} / \mathrm{cm}^{2}\right)$ areal densities with beams of $\mathrm{He}^{+}$and $\mathrm{HeH}^{+}$. In addition, we have measured the total light yield of several transitions in $\mathrm{He} \mathrm{I}, \mathrm{He} \mathrm{II}$, and $\mathrm{H}$ as a function of foil thickness using beams of $\mathrm{HeH}^{+}$ions. Experiments were done with He-foil exit energies of 125, 500, 550, and $650 \mathrm{keV}$. He I alignment decreases in all cases for the thinnest foils when molecular projectiles are used. Total light intensities generally increase with thin foils (small proton-He emergent internuclear separation), but a few decrease or are independent of foil thickness. We are able to explain several features of the alignment and intensity data in terms of the formation of quasimolecular $\mathrm{HeH}^{+}$states at or near the foil surface. Alignment reduction results from incoherent Stark mixing of the $\mathrm{He}$ I excited states in the field of the close proton. A calculation of the rms emergent $\mathrm{H}-\mathrm{He}$ internuclear separation as a function of foil thickness and beam energy is presented.
\end{abstract}

\section{INTRODUCTION}

Fast ions traversing th in solid foils emerge in a variety of excited electronic states. Those states which involve single or multiple excitation of outer-shell electrons are produced at or near the foil surface. ${ }^{1,2}$ In general, they will have nonstatistical populations within a given $n$ or $l$ manifold. In several recent experiments, we have investigated the nature of the beam-foil surface excitation process by measuring the alignment of excited states of neutral He while varying ion beam energy, foil material, and beam-current density..$^{3-6}$ One of the most interesting results of this work is that the excited-state alignment, i.e., its second moment of electron distribution, varies with the foil temperature. ${ }^{5,6}$ We have suggested that this is due directly to changes in the flux at the foil surface of slow secondary electrons created by the ion. ${ }^{2,5,6}$ There are two pieces of circumstantial evidence to support this conclusion. First, the number of secondary electrons emitted per incident ion $\gamma$ is a strong function of foil temperature.$^{5,6}$ Many of these electrons are quite close to their "parent" ions as they leave the surface. ${ }^{2,6}$ Thus, the electronic environment which the ion experiences during and just after its neutralization and excitation at the surface is a strong function of foil temperature. Secondly, there is a general correlation between the rate of change of both $\gamma$ and alignment with foil temperature; for energies where $\gamma$ is more sensitive to foil temperature, the temperature dependence of alignment is more pronounced. ${ }^{6}$ To prove that secondary electron interactions are in fact responsible for the alignment variations, it would be necessary to decouple any effects due uniquely to foil temperature from those caused by the electrons. This is difficult to do experimentally because kinetic secondary emission is only slightly dependent on foil material or surface conditions. ${ }^{7.8}$

In order to learn more about the interactions of fast atoms with correlated charged particles at the foil surface, we have used $\mathrm{HeH}^{+}$molecular ions as projectiles instead of the usual $\mathrm{He}^{+}$ions. As the molecule enters the foil, its electrons are stripped away and it dissociates, broken apart by the combined effects of internuclear Coulombic repulsion and multiple scattering from the electrons and nuclei of the target. The average distance between the proton and helium nucleus at foil exit increases monotonically with foil thickness. Thus we may vary the charge distribution about the emerging helium ion without changing foil temperature. More generally, by altering the basic foil surface-ion excitation in a relatively well-defined manner, we may hope to gain new specific information about such processes.

We report here the results of two series of experiments. First, alignments of several states of $\mathrm{HeI}$ were measured as a function of foil thickness using both $\mathrm{HeH}^{+}$and $\mathrm{He}^{+}$projectiles. In addition, total light intensities of transitions from these states as well as several others were measured using both types of projectiles, again as a function of foil thickness.

We have recently published preliminary results of these experiments. ${ }^{9}$ Other investigators have also reported the results of a similar experiment. ${ }^{10}$ While this work represents the first observation of molecular effects in beam-foil light source polarization, other molecular "cluster" effects have been observed previously. For example, Gaillard et al., ${ }^{11}$ have measured an enhanced neutral fraction for hydrogen emerging from thin carbon targets bombarded by $\mathrm{H}_{2}^{+}$and $\mathrm{H}_{3}{ }^{+}$instead of protons. Thieberger has seen 
similar effects with oxygen projectiles. ${ }^{12} \mathrm{Gab}-$ rielse has observed enhancement of normalized $\mathrm{H} \mathrm{Ly}-\alpha$ radiation using molecular hydrogen projectiles. ${ }^{13}$ These results are considered in more detail in Sec. IV.

\section{EXPERIMENT}

Beams of $\mathrm{HeH}^{+}$and $\mathrm{He}^{+}$ions were produced by a small electrostatic accelerator at the University of Chicago $(<250 \mathrm{keV})$ and the Argonne Physics Division Dynamitron ( $\geqslant 500 \mathrm{keV})$. Upon entering the target chamber, they were collimated to a diameter of $4.8 \mathrm{~mm}$. Beam-current density was held between 4 and $8 \mu \mathrm{A} / \mathrm{cm}^{2}$. Foils of varying thickness were mounted with their surface normals parallel to the beam axis on $\mathrm{Al}$ holders $1-\mathrm{mm}$ thick containing circular apertures $6.4 \mathrm{~mm}$ in diameter. Up to 23 of these could be mounted on a wheel and sequentially rotated into the beam. The beam was stopped in a Faraday cup and the current digitized, thus providing countdown pulses for preset photon counting intervals. Nominal target chamber pressure was $(7-8) \times 10^{-7}$ Torr.

Carbon foils with areal densities between 1.3 and $110 \mu \mathrm{g} / \mathrm{cm}^{2}$ were used. The thickness error was specified by the manufacturer to be $1 \mu \mathrm{g} / \mathrm{cm}^{2}$ or $10 \%$, whichever was larger. In all cases, beam energy was adjusted so that He exit energy was constant for a given series of runs. The polarization (and hence the Fano-Macek alignment parameter $A_{0}^{\text {col }}$ of the upper level) of the HeI $2 s^{1} S-3 p^{1} P(5016 \AA), 2 s^{3} S-3 p^{3} P(3889 \AA)$, $2 p^{1} P-3 d^{1} D(6678 \AA)$, and $2 p^{3} P-3 d^{3} D(5876 \AA)$ transitions were measured as described in the previous paper.

By measuring the length of the $2 s^{3} S-3 p^{3} P J, J^{\prime}$ $=2,1$ fine-structure oscillations downbeam from the foil, we were able to monitor the He exit energy to better than $4 \%$. This became important when very thick foils were used. Within experimental error, stopping powers for ${ }^{4} \mathrm{He}^{+}$on amorphous carbon were found to be given accurately by the data of Matteson et al. ${ }^{14}$ for energies above $500 \mathrm{keV}$ and by the tables of Northcliffe and Schilling for lower energies. ${ }^{15}$ No cluster effects were observed in the stopping power for molecular projectiles. Th is is not surprising at our velocities, where the dynamic screening length is $<3$ a.u.

We measured photon counts for sufficient periods of time to ensure that absolute statistical uncertainty in the experimentally measured asymmetries $(P$ or $M / I)$ would be less than $0.5 \%$. This was often difficult because of the low beam currents of $\mathrm{HeH}^{+}$produced by the accelerators. To minimize the effects of foil thickening due to ion beam bombardment (see Appendix A), runs in which foils thinner than $10 \mu \mathrm{g} / \mathrm{cm}^{2}$ were used were kept below $25 \mathrm{~min}$. Typically 20000 to 75000 counts would be collected during a given run for each orientation of the polaroid or phase plate. These elements were rotated through $360^{\circ}$ to minimize the effect of any instrumental asymmetries.

The total intensity measurements were made with exit energies of $650 \mathrm{keV}$ using a $0.75-\mathrm{m}$ Spex/Czerny-Turner monochromator to select transition wavelengths. ${ }^{2}$ The polarization elements were removed and the lens focused beam light on the monochromator's entrance slit. To avoid spurious cascading effects, measurements were made a short distance ( 1 to $2 \mathrm{~mm}$ ) downbeam from the foil, corresponding to about $3 \times 10^{-10}$ sec flight time. The beam current was measured with an empty foil holder in place. A foil was then rotated into the beam, photons were counted for a preset time, and the beam current remeasured. Light count was then normalized to average beam current. If the current fluctuated more than $5 \%$ during the run, the point was remeasured. Counting statistics for a given foil thickness were always better than $3 \%$. When a series of runs for a given transition was taken over the thickness range $2-100 \mu \mathrm{g} / \mathrm{cm}^{2}$, the beam current was not allowed to vary by more than $20 \%$. For the thickest foils, the angular half-width of the emerging He due to multiple scattering can be as large as $4^{\circ}$. (see Appendix B). ${ }^{16}$ This effect could artificially reduce the light yield if only a small horizontal section of the beam is observed. Our optics focused a vertical segment $6 \mathrm{~mm}$ in height typically $2-\mathrm{mm}$ downbeam from the foil thereby eliminating this problem. Another source of error could result from incorrect energy compensation. Our adjustment for energy loss in the foil was good to better than $10 \%$ of the total correction. The maximum adjustment was for $100 \mu \mathrm{g} / \mathrm{cm}^{2}$ foils when, for $650-\mathrm{keV}$ $\mathrm{He}$ exit energy, we used $1045-\mathrm{keV} \mathrm{HeH}{ }^{+}$. An error of $10 \%$ in the energy correction thus results in a $2 \%$ error in the He velocity. This corresponds to roughly a $2 \%$ error in the light intensity for a fixed observation position.

\section{RESULTS}

A. Alignment results

The alignment of the $3 p^{1} P, 3 p^{3} P, 3 d^{1} D$, and $3 d^{3} \mathrm{D}$ states of $\mathrm{He} \mathrm{I}$ for both $\mathrm{He}^{+}$and $\mathrm{HeH}^{+}$projectiles as a function of foil thickness are shown in Figs. 1-4. These data are tabulated in Ref. 2. The indicated errors are statistical, and do not include possible systematic effects due to 


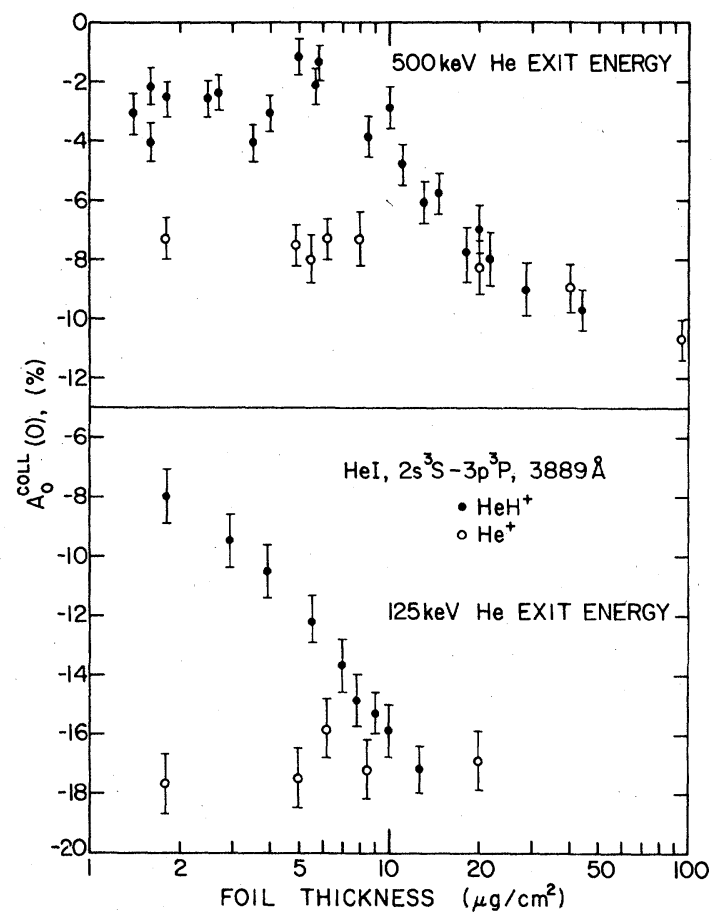

FIG. 1. He I $3 p^{3} P$ alignment vs foil thickness for $\mathrm{HeH}^{+}$ and $\mathrm{He}^{+}$beams. $550-$ and $650-\mathrm{keV}$ data is shown in $\mathrm{Fig}$. 6.

unknown foil variables. The errors include the uncertainty resulting from correction for instrumental polarization, which was never greater than $0.2 \%$ in $P$. The foil thickness errors bars are not shown. The correspondence between foil thickness and the emergent $\mathrm{rms} \mathrm{He}-\mathrm{H}$ internuclear separation for two different He exit energies is shown in Fig. 5. The calculation leading to these curves is given in Appendix B. It includes the contributions of multiple scattering from both nuclei and electrons, Coulomb explosion, and the different rates of energy loss for the proton and He nucleus.

With $\mathrm{HeH}^{+}$projectiles, the magnitude of alignment is reduced by at least a factor of 2 for the thinnest foils. The fractional reduction is generally greater for $D$ than for $P$ states. At 650 $\mathrm{keV}-\mathrm{He}$ exit energy, the $3 d^{3} D$ alignment is es sentially zero for foils thinner than $20 \mu \mathrm{g} / \mathrm{cm}^{2}$. For high energies, excepting the $3 p^{1} P$ data, alignment is insensitive to the proton distribution for thicknesses below $10 \mu \mathrm{g} / \mathrm{cm}^{2}$. At low energy the "equilibrium thickness," i.e., the thickness at which $\mathrm{HeH}^{+}$values begin to equal those for atomic projectiles is, within experimental error, independent of the excited state involved. Equilibrium occurs at $10 \pm 4 \mu \mathrm{g} / \mathrm{cm}^{2}$, corresponding to average emergent internuclear separations of $30 \pm 10$ a.u. In contrast, at high energy, the ${ }^{3} D$

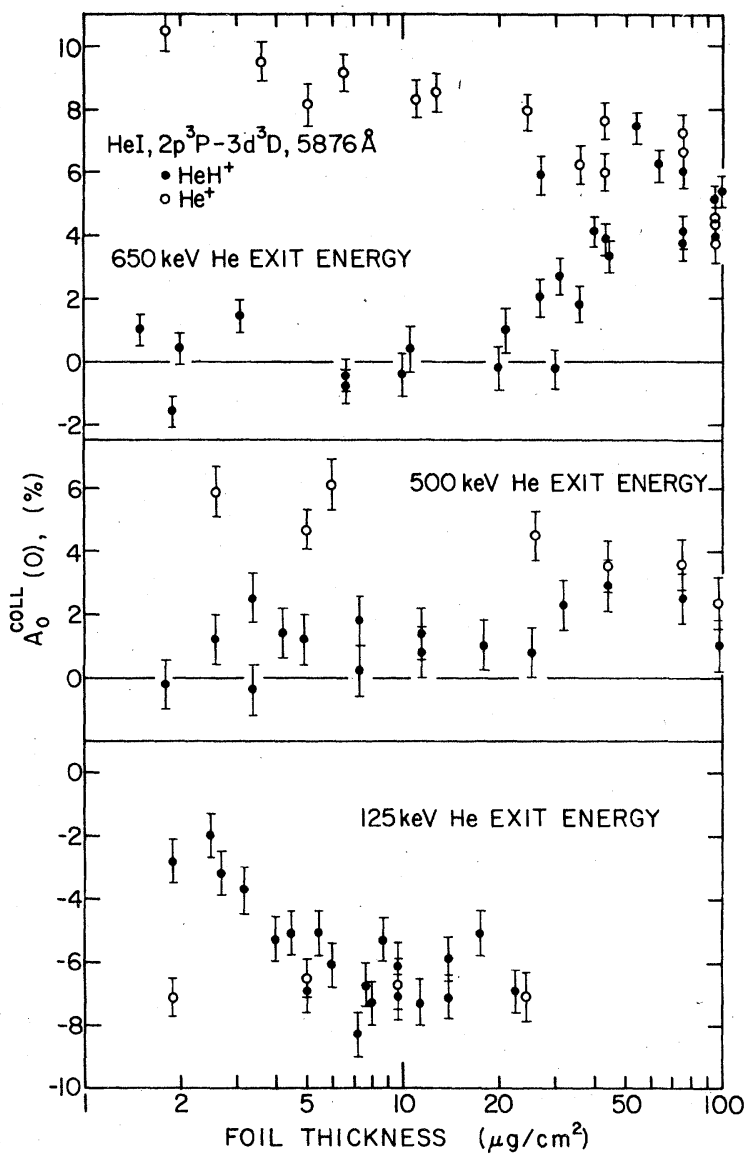

FIG. 2. He I $3 d^{3} D$ alignment vs foil thickness for $\mathrm{HeH}^{+}$ and $\mathrm{He}^{+}$beams.

state requires conside rably thicker foils to reach equilibrium than does the ${ }^{3} P$ state.

All $3 p^{3} P \mathrm{HeH}^{+}$results have been combined in Fig. 6 by plotting the alignment normalized to the $\mathrm{He}^{+}$thin-foil value as a function of emergent internuclear separation. Data taken for four different He exit energies coalesce fairly well on a universal curve. While the re appears to be a slight systematic difference between the $125-\mathrm{keV}$ points and the other data, it is not statistically significant. The $\mathrm{HeH}^{+}$data reach the atomic thinfoil value at $35 \pm 15 \mathrm{a}$.u. The $3 d^{3} D$ results do not scale similarly, as can be seen in Fig. 2.

Figure 6 accentuates another interesting, general feature of the data. For very thick $(>20$ $\mu \mathrm{g} / \mathrm{cm}^{2}$ ) foils, $A_{0}^{\text {col }}(0)$ decreases for both atomic and molecular projectiles. This thickness dependence is marked for the high-energy results, but is not seen in the $125-\mathrm{keV}$ data (with the exception of the $3 p^{1} P$ alignment), presumably because of the reduced thickness range at this energy. This effect is almost certainly due to increasing foil temperature,$^{5,6}$ but attempts to prove 


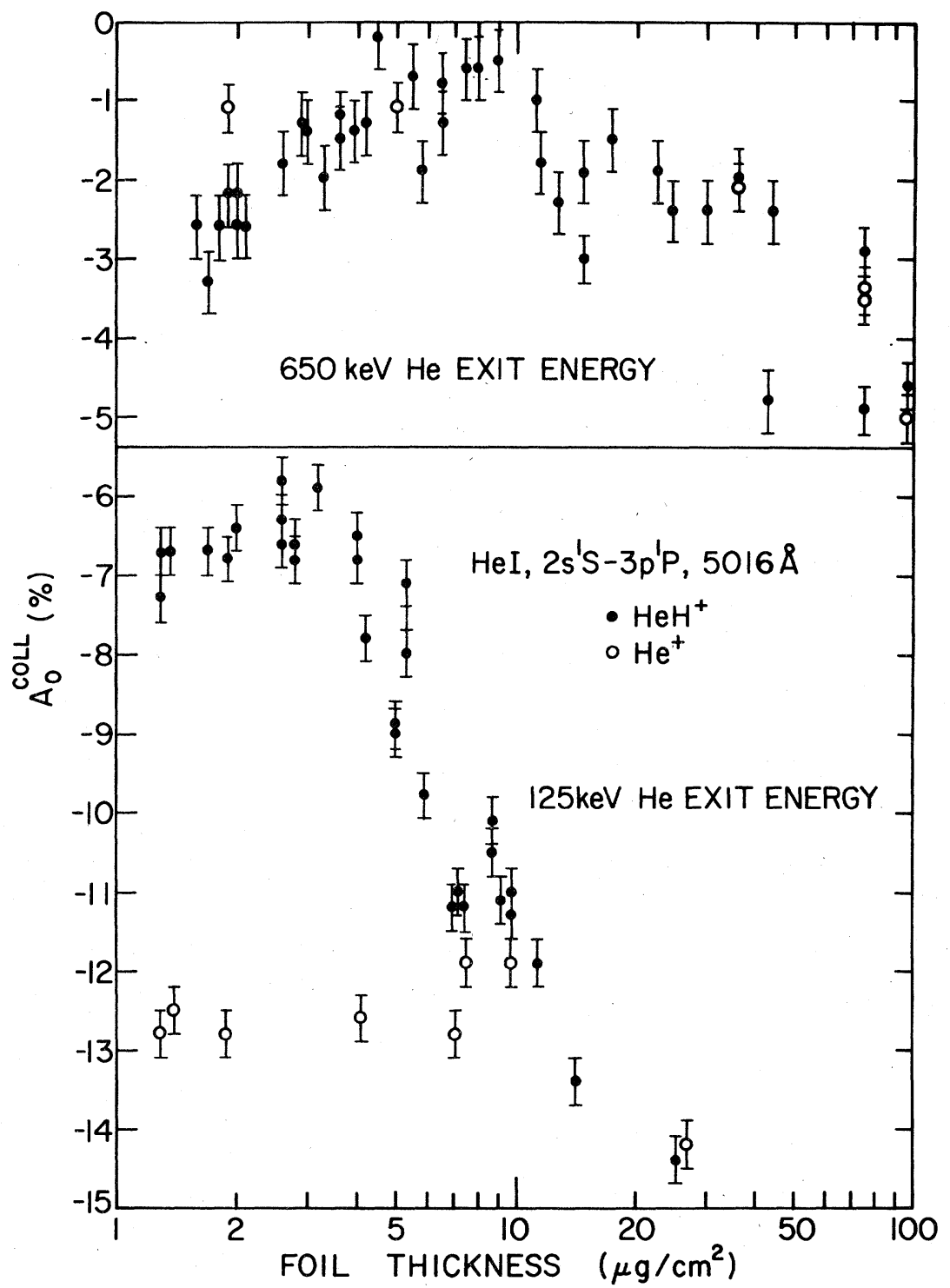

FIG. 3. He I $3 p^{1} P$ alignment vs foil thickness for $\mathrm{HeH}^{+}$and $\mathrm{He}^{+}$beams. Note decrease of $A_{0}^{\text {col }}$ for thinnest foils, $\mathrm{HeH}^{+}$ incident.

this have been inconclusive. We have measured the foil temperature to increase with thickness for fixed beam current, but the increase is not large enough $(\sim 125 \mathrm{~K})$ to expla in the entire alignment variation. It is hard to envision systematic effects which could produce this result. In all likelihood, the temperature measurement, and more specifically the estimate of thick-foil emissivity (extrapolated from earlier measurements below $\left.40 \mu \mathrm{g} / \mathrm{cm}^{2}\right),{ }^{5}$ is in error.

The ${ }^{1} P$ and ${ }^{3} P$ data are qualitatively different in two respects. First, the atomic projectile alignment values are different. This has been shown to be true over a broad range of energies, and results from the $P$ state spin dependence of the beam-foil excitation process. ${ }^{\circ}$ Secondly, the $\mathrm{HeH}^{+}$projectile $3 p^{1} P$ alignment is increasingly negative for the thinnest foils. This is seen clearly at $650 \mathrm{keV}$ and to a lesser extent at 125 $\mathrm{keV}$. The equivalent $3 p^{3} P$ data is independent of thickness over the same range at $650 \mathrm{keV}$. This difference, as we shall see, results from the formation of quasimolecular $\mathrm{HeH}^{+}$orbitals near the foil's exit surface.

The equilibrium thickness $T_{1}$ and $T_{2}$, the thickness at which the alignment for incident $\mathrm{HeH}^{+}$ is halfway between its thin foil and equilibrium values, are listed with the corresponding rms internuclear separations $L_{1}$ and $L_{2}$ in Table $I$. The actual distribution of the emergent separa- 


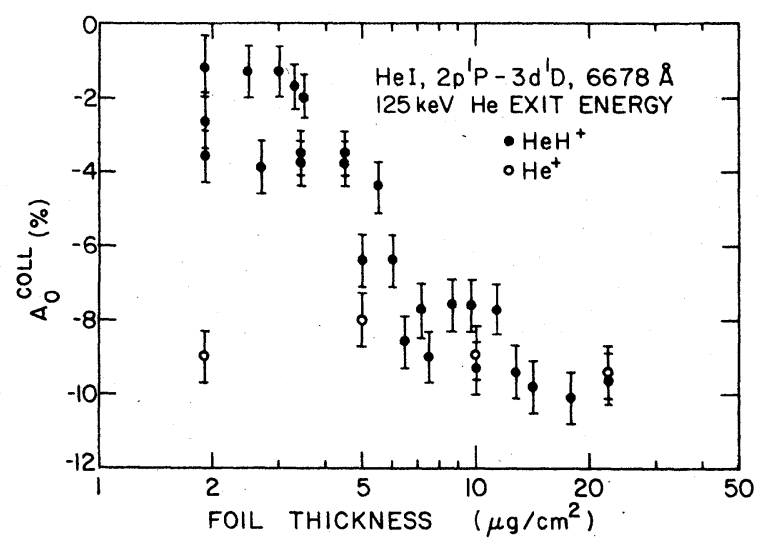

FIG. 4. He I $3 d^{1} D$ alignment vs foil thickness for $\mathrm{HeH}^{+}$ and $\mathrm{He}^{+}$beams.

tions for a given foil thickness above $\sim 10$ $\mu \mathrm{g} / \mathrm{cm}^{2}$ can be quite broad (see Appendix B). Th is means that in assigning characteristic internuclear separations to features of the data, we must keep in mind that they are averages of the entire distribution and as such represent upper limits to the length scale for specific processes. Thus $L_{2}$ is probably a better estimate of the length

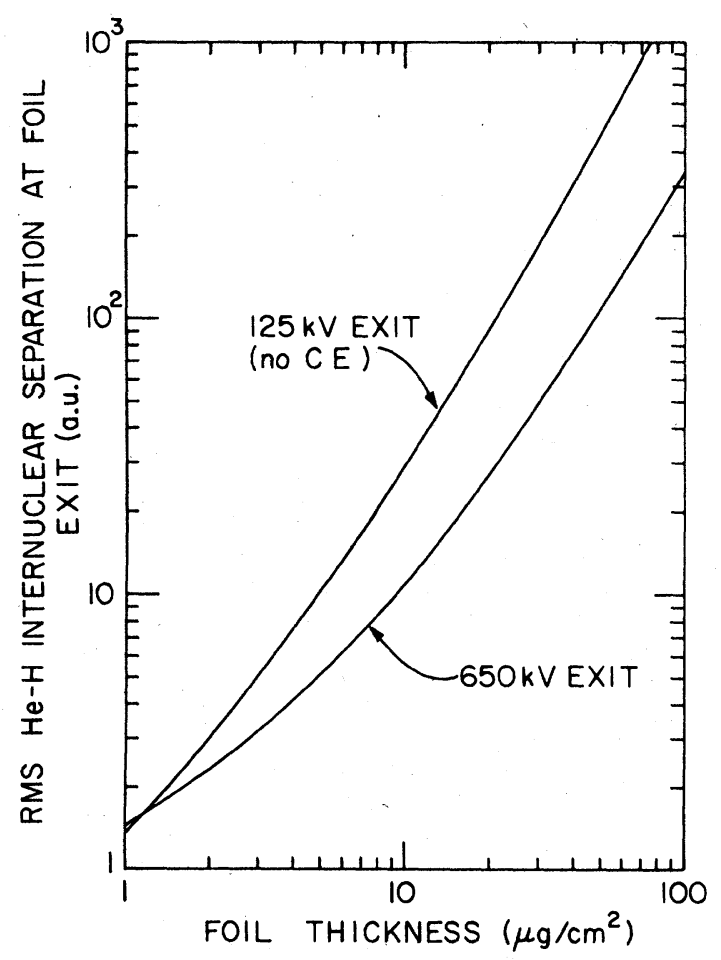

FIG. 5. Calculated $\mathrm{He}-\mathrm{H} \mathrm{rms}$ emergent internuclear separation for $125-$ and $650-\mathrm{keV}-\mathrm{He}$ exit energy. Coulomb explosion contributions to the $125-\mathrm{keV}$ curve are negligible. See Appendix B for details of the calculation.

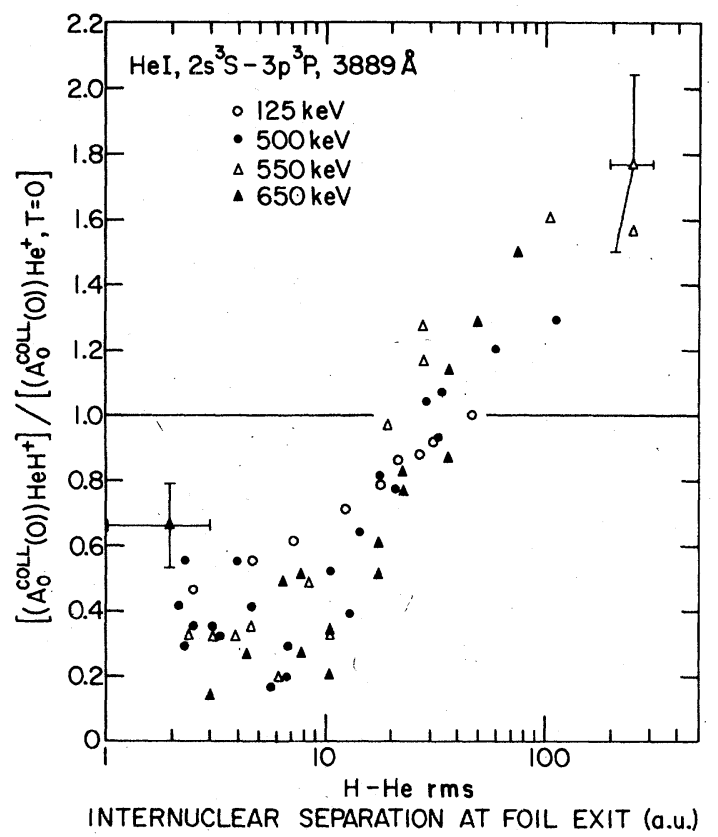

FIG. 6. HeI $3 p^{3} P \mathrm{HeH}^{+}-\mathrm{He}^{+}$alignment ratio vs $\mathrm{rms}$ internuclear separation at foil exit. The ordinate is the ratio of the alignment for $\mathrm{HeH}^{+}$beams to that for $\mathrm{He}^{+}$ beams with thin foils. Typical error bars shown.

scale for the destruction of alignment by the close proton. We also list $\eta$, the ratio of the thin-foil $\mathrm{HeH}^{+}$alignment to the thin-foil atomic value.

\section{B. Total light yield results}

Total relative light yield (which is proportional to upper-state population) as a function of foil thickness for the $3 p^{1} P$ and $3 d^{1} D$ states at 650 keV He exit energy are shown in Figs. 7 and 8. We have already published results for the $n=3$ triplet states. ${ }^{9}$ All intensities are normalized to the average intensity measured for the thickest foils in each set. The large scatter of the data is nonstatistical and is not understood. Note that scatter in alignment data for the same states is statistical.

We have measured several other lines in neutral helium as well as in $\mathrm{HeII}$ and $\mathrm{H}$. These results, all taken with $\mathrm{HeH}^{+}$projectiles and a $\mathrm{He}$ exit energy of $650 \mathrm{keV}$ are shown in Fig. 9. The $D$ state and hydrogenic transitions show strong variations over the thickness range, with $n=3$ transitions having higher. relative intensities for thin foils than do their $n=4$ counterparts. The $P$-state intensities exhibit less variation; the $n=3$ data is independent of thickness above $8-9 \mu \mathrm{g} / \mathrm{cm}^{2}$. The $2 s^{3} S-5 p^{3} P$ intensity actually decreases for thin foils. Measurements of the $2 s^{3} S-4 p{ }^{3} P$ transition, taken at a different energy, also show a decrease from the equilibrium 
TABLE I. Equilibrium thicknesses and extinction ratios for He I (see text).

\begin{tabular}{ccccccc}
\hline \hline Excited state & He exit energy & $T_{1}\left(\mu \mathrm{g} / \mathrm{cm}^{2}\right)$ & $L_{1}$ (a.u.) & $T_{2}\left(\mu \mathrm{g} / \mathrm{cm}^{2}\right)$ & $L_{2}$ (a.u.) & $\eta$ \\
\hline $3 p^{3} P$ & 125 & $12(2)$ & $39(10)$ & $6(1)$ & $13(3)$ & $0.48(0.05)$ \\
& 500 & $21(3)$ & $35(6)$ & $11(2)$ & $15(4)$ & $0.37(0.10)$ \\
& 550 & $17(3)$ & $25(6)$ & $11(2)$ & $14(3)$ & $0.32(0.10)$ \\
$3 p^{1} P$ & 650 & $24(3)$ & $34(5)$ & $16(2)$ & $19(4)$ & $0.33(0.10)$ \\
$3 d^{3} D$ & 125 & $11(2)$ & $34(10)$ & $7(1)$ & $17(3)$ & $0.52(0.05)$ \\
& 125 & $8(2)$ & $21(7)$ & $4(1)$ & $7(3)$ & $0.24(0.10)$ \\
$3 d^{1} D$ & 550 & $55(10)$ & $150(40)$ & $35(10)$ & $75(30)$ & $0.20(0.05)$ \\
& 650 & $70(20)$ & $180(80)$ & $40(10)$ & $75(25)$ & 0 \\
\hline \hline
\end{tabular}

value. The $2 s^{1} S-3 p^{1} P$ intensity curve is interesting in that it falls much more quickly to its equilibrium value than do other transitions which exhibit variations with thickness. This anomalous behavior is complimented by the pronounced decrease of $A_{0}^{\text {col }}$ for the $3 p^{1} P$ state in the same thickness range.

\section{DISCUSSION}

We first discuss the total light intensity data in terms of cluster effects and a molecular model. Cluster effect arguments have been used previously to explain molecular beam-foil chargestate measurements. Gaillard et al. ${ }^{11}$ measured the neutral fraction of hydrogen emerging from thin carbon foils bombarded by $\mathrm{H}^{+}, \mathrm{H}_{2}{ }^{+}$, and $\mathrm{H}_{3}{ }^{+}$. These results are shown graphically in Fig. 7 of Ref. 11. Note that for all emergent internuclear separations, the normalized neutral production has the ratio $(1+2 k) /(1+k) / 1$ for $\mathrm{H}_{3}{ }^{+}$,

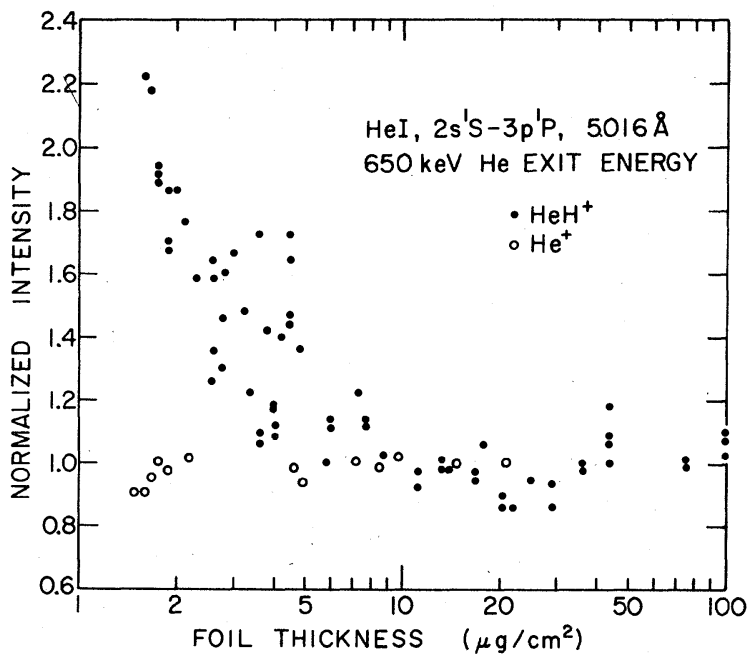

FIG. 7. Total relative intensity of the $2 s^{1} s-3 p^{1} P 5016-$ $\AA$ transition vs foil thickness. Typical statistical error is $1-3 \%$.
$\mathrm{H}_{2}{ }^{+}$, and $\mathrm{H}^{+}$, respectively, where $k$ decreases with increasing separation. Gaillard et al. have proposed a simple model to expla in these results. As a proton emerges from the foil there is some probability $P$ that it will be neutralized. This probability is independent of the proximity of the other protons. In addition, there is a smaller probability $P^{\prime}$ that the proton will capture an electron correlated with another dissociated proton. The ratio of neutrals for $\mathrm{H}_{3}^{+}, \mathrm{H}_{2}{ }^{+}$, and $\mathrm{H}^{+}$projectiles will thus be $\left[1+\left(2 P^{\prime} / P\right)\right] /\left[1+\left(P^{\prime} / P\right)\right] / 1$. Since $P^{\prime}$ decreases with increasing proton-proton separation, the results a re qualitatively explained by this model. The cluster effect disappears for emergent separations greater than $\sim 30$ a.u. Gabrielse has observed a similar $\mathrm{H} \mathrm{Ly}-\alpha$ intensity enhancement over the $\mathrm{H}^{+}$projectile case for $\mathrm{H}_{2}{ }^{+}$and $\mathrm{H}_{3}{ }^{+} \cdot{ }^{13}$ However, he measures an equilibrium separation of 15 a.u. and his value for $k$, extrapolated to zero foil thickness, is approximately one, as opposed to the Gaillard et al. value of 0.5 . Finally, Thieberger has measured the charge-state distribution of oxygen ions resulting from $\mathrm{O}_{2}^{-}$and $\mathrm{O}$ bombardment of carbon foils. ${ }^{12}$ As seen for hydrogen, the average atomic charge fraction is reduced when $\mathrm{O}_{2}^{-}$projectiles are used.

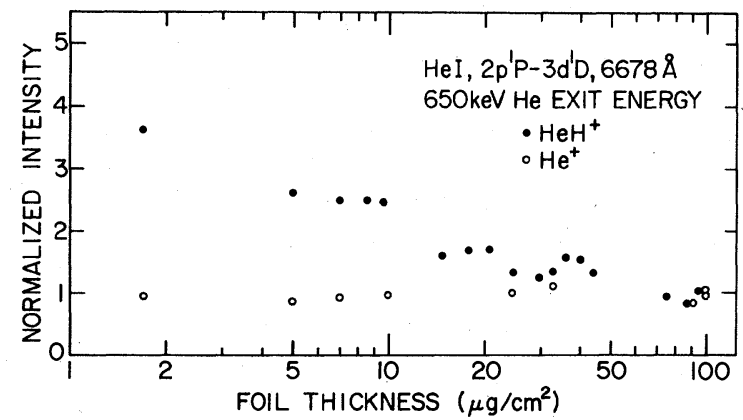

FIG. 8. Total relative intensity of the $2 p^{1} P-3 d^{1} D$ $6678 \AA$ transition vs foil thickness. Statistical error is $<2 \%$. 


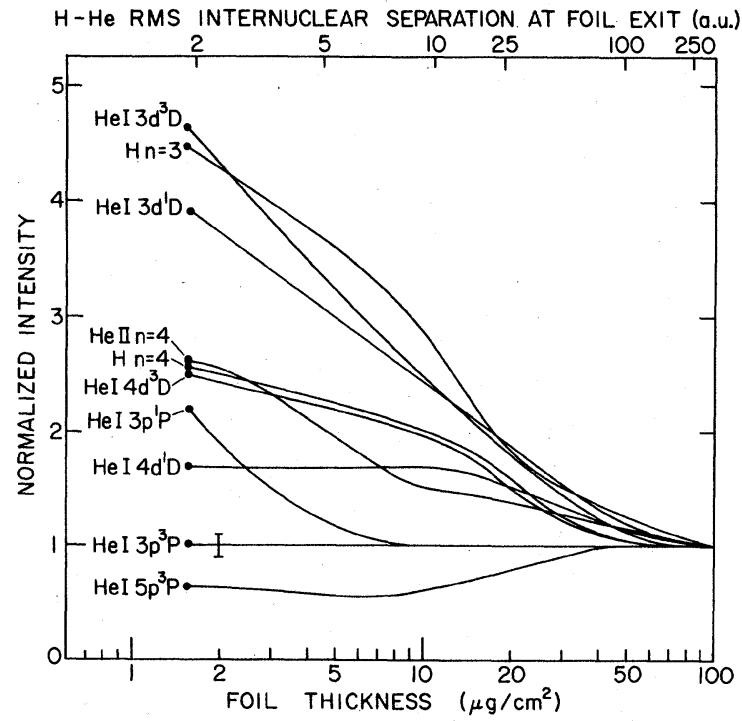

FIG. 9. Total relative intensity of several transitions in HeI, HeII, and $\mathrm{H}$. He I and $\mathrm{H}$ transitions are to $n=2$ levels. The He II transition is $n=4-3$. Data smoothed by eye. All intensities are normalized to the equilibrium value. Vertical bar indicates typical data scatter. $\mathrm{HeH}^{+}$ incident, $650-\mathrm{keV}-\mathrm{He}$ exit energy. Total intensity for transitions shown with $\mathrm{He}^{+}$incident was independent of foil thickness.

All of these effects can be understood in terms of the cluster model presented above. Much of our intensity data can be understood in this manner as well. A simple decrease of the average $\mathrm{H}$ and $\mathrm{He}$ charge fractions, with different values of $P^{\prime}$ for the various $n$ levels, would qualitatively explain the $D$ state and hydrogenic intensity enhancements. Such considerations fail, however, to account for many features of our intensity curves. The $n=3,4$, and $5{ }^{3} P$ state populations are either unaffected or decrease under the influence of a close proton. HeI $P$ and $D$ states behave quite differently over the entire thickness range. The $3 P$ data exhibit a strong spin dependence.

Thus the correlated electron cluster effect model needs to be improved to include the dependence of electron capture (or loss) probability on the excited states involved. For small internuclear separations, where cluster effects are greatest, the formation of quasimolecular states must be considered. Singlet-triplet differences, for example, which a re not explicitly included in a first-order cluster effect model, become important when we consider molecular state formation at the foil surface.

The three molecules which could dissociate to produce neutral helium are $\mathrm{HeH}^{-}, \mathrm{HeH}$, and $\mathrm{HeH}^{+}$. Assuming, as a first approximation, that the charge state of $\mathrm{H}$ and $\mathrm{He}$ are determined independently, at 650-keV-He exit energy the relative probability for the two nuclei to share 2,3 , or 4 electrons is 32 to 1 to $0.02 .^{17}$ Thus we consider $\mathrm{HeH}^{+}$with regard to the HeI data. Higher charge states of the $\mathrm{He}-\mathrm{H}$ complex become important when dealing with the hydrogenic results.

Green et al..$^{18-21}$ have made $a b$ initio calculations of the ground and first 40 excited states of $\mathrm{HeH}^{+}$. The correlation diagrams for these states are graphed in Refs. 18, 20, and 21. The uppermost excited states they consider are those which go to $n=4$ states of $\mathrm{H}$ and $\mathrm{He}$ in the separated atom limit. For $R$ (internuclear separation) greater than 1.5 a.u., $\mathrm{HeH}^{+}$molecular orbitals are very similar to those of $\mathrm{H}_{2}{ }^{+} \cdot{ }^{18}$ However, states of a given symmetry $(\Sigma, \mathrm{II}, \Delta)$ avoid crossing each other, primarily because of core-penetration effects. ${ }^{22}$ If the molecule, as it is dissociating, passes through the region of an avoided crossing with sufficient velocity, it can effectively jump from one state to the other "diabatically." diabatic behavior is

$$
v / \lambda \gtrsim \Delta E,
$$

which is simply a statement of the condition that the uncertainty in the electronic energy of the molecule be greater than the energy diffe rence $\Delta E$, between the two levels. The width of the crossing region is denoted by $\lambda$. Landau-Zener theory approximates the avoided crossing as the interaction of two potential curves and gives the probability for the system to make a transition from one state to the other.

Even for the thinnest foils (low internuclear velocities), we are in the diabatic region. The $\mathrm{He}-\mathrm{H}$ emergent separation velocity with $650 \mathrm{-keV}-$ He exit energy and a $2-\mu \mathrm{g} / \mathrm{cm}^{2}$ foil is 0.02 a.u. (see Table III). Assuming $\lambda=2$ a.u. (see, e.g., Fig, 2 of Ref. 18) diabatic crossings will occur when minimum energy separations are of the order of 0.01 a.u. This is the case for virtually all of the avoided crossings in $\mathrm{HeH}^{+}$.

The observed thickness-dependent intensity variations may be understood in terms of an initial molecular state with a relatively large population feeding, via diabatic crossing, a less populated final state. Such a mechanism for a two-level system is shown schematically in Fig. 10. Molecular orbitals which correlate to different $n$ levels in the united atom limit will have populations which may be expected ${ }^{24,25}$ to vary roughly as $n^{-3}$. Thus in Fig. 10, the separated atom $n=3$ level population will increase for the thinnest foils, whereas that of the $n=2$ level will decrease. Variations of this sort will occur, 


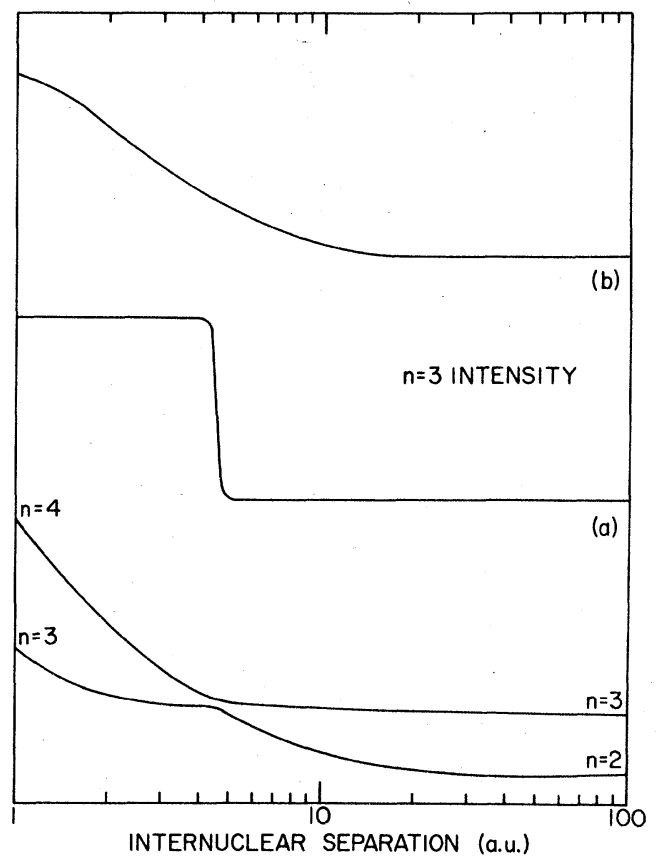

FIG. 10. Schematic showing the effect of molecular diabatic level crossing on intensity. Two levels [similar to the $(e, E)$ and $(f, F) \Sigma$ states of $\left.\mathrm{HeH}^{+}\right]$which in the adiabatic limit avoid crossing are shown. The lower curve, populated more strongly than the upper level, feeds the final $n=3$ state through diabatic crossings. When the emergent internuclear separation is a radial delta function, $n=3$ intensity varies as in (a). The distributional width of the internuclear separation modifies this variation to look like (b).

of course, only if the re is a reasonable probability of a diabatic crossing between the two states. The effect of distributional smearing is shown in the upper curve of Fig. 10. The internuclear separations at which the diabatic crossings occur vary, as we would expect, from state to state (see Fig. 9). The HeI $4 d^{1} D$ curve appears to result from a single crossing at $\sim 25$ a.u., while the $3 p^{1} P$ crossing occurs closer in, at $\sim 5$ a.u. The HeII $n=4$ curve may involve two transitions at different $R$.

We consider as an example the HeI $3 P$ states. The $3 p^{3} P(3889 \AA)$ intensity is independent of thickness, suggesting that after formation of the molecular $\mathrm{HeH}^{+}$state to which it correlates, repopulation due to level crossing is negligible. The $3 p^{1} P$ state, on the other hand, appears to be repopulated by another state for $R \approx 5$ a.u. Crossings between states of different symmetry are virtually the same for singlets and triplets..$^{2,18-21}$ Avoided crossings for states of the same symmetry, however, are considerably different. These differences occur primarily below 1 a.u. (due to the large singlet-triplet variation in
${ }^{5} \mathrm{Li}^{+}$) but are present in the $\Sigma$ states even at large $R .^{21}$

The $F^{1} \Sigma$ state (fifth from the bottom in Fig. 2 of Ref. 18) has well-defined avoided crossings with the $E^{1} \Sigma$ and $G^{1} \Sigma$ states at 7.0 and 16.1 a.u., respectively. ${ }^{26}$ Its triplet equivalent (see Fig. 1, Ref. 21) has a less strongly avoided crossing at 15.2 a.u. with the next higher level. ${ }^{26}$ There is a weak interaction with the next lower state at roughly 7 a.u. The $F^{1} \Sigma$ and $f^{3} \Sigma$ states correspond to the HeI $3 P$ state in the separated atom limit assuming diabatic behavior for $R>10 \mathrm{a} . \mathrm{u}$. In the region around $10 \mathrm{a} . \mathrm{u}_{\text {., }}$ they are similar to the $\mathrm{H}_{2}{ }^{+} 4 d \sigma \mathrm{MO}$ (molecular orbit). The $(e, E)$ states, correlating to a ${ }^{5} \mathrm{Li}^{+} n=3$ level in the united atom limit will have larger initial populations than the $(f, F)$ states, which are promoted to $n=4$. The $(e, E)$ levels are like the $4 d \sigma \mathrm{MO}$ before the avoided crossing at 7 a.u., and are similar to the $4 f \sigma \mathrm{MO}$ following it. The probability of making a jump from one state to another is given by Landau-Zener theory to be

$$
P_{a \rightarrow b}=\exp \left[\left(-\pi \Delta E^{2}\right) /\left(2 v\left|d / d R\left(E_{a}-E_{b}\right)\right|_{0}\right)\right],
$$

where $v$ is the internuclear velocity and the subscript zero indicates that the radial derivatives refer to the unperturbed energy curves. ${ }^{27}$ The minimum energy separations for the avoided crossing at 7 a.u. are 0.006 and 0.019 a.u. for the singlet and triplet states, respectively. ${ }^{26} \mathrm{As}-$ suming an internuclear velocity of 0.02 and using the radial derivatives of the $4 f \sigma$ and $4 d \sigma \mathrm{H}_{2}{ }^{+}$ MO's, the transition probability between the $(e, E)$ and $(f, F)$ levels is 0.86 for the singlets and 0.20 for the triplets. $F^{1} \Sigma$ states of quasimolecules with initial internuclear separations less than 7 a.u. will thus be populated more than $f^{3} \Sigma$ levels, leading to a relative enhancement of the $3 p^{1} P$ population. While we would expect a slight increase in $2 s^{3} S-3 P^{3} P 3889-\AA$ intensity for thin foils from these considerations, other factors may affect the final atomic states. The qualitative features of the $3 P$-state data are explained by this model.

Similar mechanisms are presumably responsible for the general intensity enhancement of the HeI $D$ states and the hydrogenic transitions. Highly promoted orbitals ( $4 f \sigma, 5 f \sigma$, etc., see Fig. 3 of Ref. 22) correlating to separated atomic states with principal quantum number $n_{s}$ will be fed by MO's connected to united atom states with $n_{u}=n_{s}+\Delta$ where $\Delta \geqslant 0$. Initial populations of these states will vary as $n_{u}^{-3}$. The $n_{s}=3$ and $4 D$ states are thus fed predominantly by $n_{u}=4$ and 5 levels, respectively. The expected enhancement is thus $\left(\frac{5}{4}\right)^{3}=1.95$, close to what we measure.

There are large rotational coupling matrix 
elements between 2 and 3 a.u. for $\mathrm{HeH}^{+}$states corresponding diabatically to the $\mathrm{H}_{2}{ }^{+}, 4 f \pi, 4 f \delta$, $4 d \sigma$, and $4 d \pi$ MO's $(\Delta m= \pm 1){ }^{26}$ In the separated atom limit, the $4 f$ orbitals correspond to $D$ states, and the $4 d$ orbitals to $P$ states. If the $4 d$ orbitals had much higher initial polarization than the $4 f$ 's, the large variation with thickness of the $D$ states relative to that of the $P$ states (at least for $n=3$ ) could be explained. It seems unlikely, however, that this is the case. As it stands, this feature of the data remains a puzzle.

We turn now to a discussion of the alignment results and first propose a qualitative model for alignment reduction by the close, dissociated proton. The distribution of this proton about the emerging He atom is not isotropic because of the differential energy loss and unequal contributions of multiple scattering from the foil electrons and nuclei, which lead to perpendicular spatial distributions (see Appendix B). The post-foil geometry thus retains a cylindrical symmetry. The electric field of the close proton creates strong Stark mixing in the He atom. Any alignment along the internuclear axis induced by this interaction will vary in time periodically with a final value dependent on some value of the phase integral $\phi$ given by

$$
\phi=C \bar{E}\left(d_{0}-D\right) / v
$$

where $C$ is the field-independent part of the Stark matrix elements under consideration, $\bar{E}$ is the average electric field along the internuclear axis during the interaction, $v$ is the internuclear velocity, $d_{0}$ is the emergent internuclear separation, and $D$ is the distance at which the Stark interaction is negligible. ${ }^{2,28}$ For a given proton orientation, the ensemble average of the final-state alignment will be zero if typical values of $\phi$ are large compared to $2 \pi$. In this case the He alignment averaged over all proton orientations must go to zero, even though the collision geometry is not completely symmetric. We may estimate the foil thickness at which alignment reduction will become significant if we assume that electric field contributions to $\phi$ a re negligible when Stark mixing is in the quadratic region. This is true for HeI $n=3$ states when $E<5 \times 10^{6} \mathrm{~V} / \mathrm{cm}$ $\left(10^{-3}\right.$ a.u.). Thus we set $D=32$ a.u. The constant $C$ is approximately equal to $2 \pi$ times the radius of the $n=3$ states, i.e., $C \approx 30$ a.u. Calculating $\bar{E}$, we may write

$$
\phi=\frac{C e^{2}}{v}\left(\frac{1}{d_{0}}-\frac{1}{D}\right) .
$$

Using Tables II and III, we estimate that alignment reduction will begin to occur $(\phi \sim 2 \pi)$ for emergent internuclear separations of 20-25 a.u. for both 125 and 650-keV-He exit energy. Owing. to distributional "smearing," values of $L_{2}$ (see Table I) are probably the best experimental de-

TABLE II. RMS H-He distance (a.u.) at foil exit vs foil thickness $\left(\mu \mathrm{g} / \mathrm{cm}^{2}\right)$. Note: Coulomb explosion is considered to be negligible for $125-\mathrm{keV}$ exit energy. CE contribution is replaced by molecular ground-state internuclear separation.

\begin{tabular}{crrrrrr}
\hline \hline He exit energy & \multicolumn{1}{c}{$t$} & $\langle d\rangle(\mathrm{CE})^{\mathrm{a}}$ & $\langle d\rangle(\mathrm{EL})^{\mathrm{b}}$ & $\left\langle d^{2}\right\rangle^{1 / 2}(\mathrm{ES})^{\mathrm{c}}$ & $\langle d\rangle(\mathrm{AS})^{\mathrm{d}}$ & \multicolumn{1}{c}{$\left(\Sigma\langle d\rangle^{2}\right)^{1 / 2}$} \\
\hline \multirow{2}{*}{$125 \mathrm{keV}$} & 2 & 1.40 & 0.49 & 1.35 & 2.08 & 2.53 \\
& 4 & 1.40 & 2.03 & 3.82 & 6.00 & 7.39 \\
& 6 & 1.40 & 4.55 & 7.02 & 11.30 & 14.10 \\
& 8 & 1.40 & 7.93 & 10.80 & 17.60 & 22.10 \\
& 10 & 1.40 & 12.40 & 15.10 & 25.20 & 31.90 \\
& 16 & 1.40 & 30.80 & 30.50 & 53.80 & 69.10 \\
& 23 & 1.40 & 60.60 & 52.50 & 97.80 & 127.00 \\
& 30 & 1.40 & 107.00 & 78.00 & 152.00 & 202.00 \\
& 2 & 2.28 & 0.07 & 0.47 & 0.40 & 2.36 \\
& $450 \mathrm{keV}$ & 3.82 & 0.27 & 1.32 & 1.15 & 4.21 \\
& 6 & 5.36 & 0.61 & 2.42 & 2.18 & 6.30 \\
& 8 & 6.89 & 1.07 & 3.71 & 3.44 & 8.61 \\
& 10 & 8.42 & 1.67 & 5.19 & 4.93 & 11.20 \\
& 15 & 12.20 & 3.74 & 9.49 & 9.55 & 18.60 \\
& 20 & 16.00 & 6.61 & 14.60 & 15.40 & 27.40 \\
& 30 & 23.60 & 14.60 & 26.60 & 30.60 & 49.20 \\
& 50 & 37.40 & 39.90 & 56.50 & 74.20 & 108.00 \\
& 100 & 75.50 & 152.00 & 155.00 & 251.00 & 340.00 \\
\hline \hline
\end{tabular}

${ }^{\mathrm{a}} \mathrm{CE}$ is Coulomb explosion.

${ }^{b} E L$ is differential energy loss.

${ }^{c}$ ES is energy straggling.

d AS is angular multiple scattering. 
TABLE III. Average center-of-mass energy (a.u.) vs foil thickness [in $\left.\left(\mu \mathrm{g} / \mathrm{cm}^{2}\right)\right]$.

\begin{tabular}{lrrrrrr}
\hline $\begin{array}{l}\text { He exit } \\
\text { energy }\end{array}$ & \multicolumn{1}{c}{$t$} & $\left(_{\mathrm{CE}}\right)^{\mathrm{a}}$ & $(\mathrm{EL})^{\mathrm{b}}$ & $(\mathrm{ES})^{\mathrm{c}}$ & $(\mathrm{AS})^{\mathrm{d}}$ & $\begin{array}{c}\text { Total } \\
E(\mathrm{~cm})\end{array}$ \\
\hline $125 \mathrm{keV}$ & 2 & & 0.11 & 0.19 & 0.23 & 0.53 \\
& 4 & & 0.49 & 0.38 & 0.51 & 1.38 \\
& 6 & & 1.10 & 0.57 & 0.82 & 2.49 \\
& 8 & & 1.90 & 0.76 & 1.16 & 3.82 \\
& 10 & & 2.99 & 0.96 & 1.55 & 5.50 \\
& 16 & & 7.52 & 1.58 & 2.86 & 12.00 \\
& 23 & & 14.80 & 2.34 & 4.67 & 21.80 \\
& 30 & & 28.40 & 3.12 & 6.74 & 38.20 \\
$55 \mathrm{keV}$ & 2 & 0.27 & 0.01 & 0.11 & 0.05 & 0.44 \\
& 4 & 0.28 & 0.04 & 0.23 & 0.10 & 0.65 \\
& 6 & 0.28 & 0.10 & 0.34 & 0.16 & 0.88 \\
& 8 & 0.28 & 0.18 & 0.46 & 0.24 & 1.16 \\
& 10 & 0.28 & 0.27 & 0.57 & 0.32 & 1.44 \\
& 15 & 0.28 & 0.62 & 0.86 & 0.55 & 2.31 \\
& 20 & 0.29 & 1.10 & 1.15 & 0.84 & 3.38 \\
& 30 & 0.29 & 2.50 & 1.73 & 1.56 & 6.08 \\
& 50 & 0.30 & 6.90 & 2.90 & 3.49 & 13.60 \\
& 100 & 0.31 & 28.30 & 5.82 & 10.55 & 44.70 \\
\hline \hline
\end{tabular}

${ }^{\mathrm{a}} \mathrm{CE}$ is Coulomb explosion.

${ }^{b} E L$ is differential energy loss.

${ }^{c} \mathrm{ES}$ is energy straggling.

${ }^{\mathrm{d}} \mathrm{AS}$ is angular multiple scattering.

termination of $d_{0}$ when $\phi$ is of the order of $2 \pi$. Thus our estimates are a bit high. Considering the crude nature of the model, this is not unexpected. The important point here is that $\mathrm{He}$ alignment reduction by the close proton results not from a more symmetric collision geometry (spherical as opposed to cylindrical), but from the length of the proton-helium interaction. In other words alignment reduction is due to a temporal averaging process instead of a spatial one.

The difference between $P$ - and $D$-state alignment thickness variations may be understood in terms of molecular considerations already discussed. At high energy, the equilibrium thickness for $D$-state alignment is considerably larger than that for the $P$ state. In addition, the $D$-state intensity and alignment reach their equilibrium values for the same foil thickness. If we assume that the strong $D$-state intensity enhancement results from a level crossing at $R<20-25$ a.u. (i.e., the critical value of $d_{0}$ for Stark alignment reduction), states populated by this mechanism will have reduced values of alignment. Alignment measured in the thickness range where intensity is enhanced will be reduced accordingly. As a result, $T_{1}$ for the $D$-states will be highly sensitive to the distribution of emergent internuclear separations and will not scale directly with a critical value of $d_{0}$ for alignment reduction. The $P$ states, which from our total intensity measurements appear to be less affected by level-crossing repopulations, will exhibit such scaling, as is seen in Fig. 6 . The strong enhancement of unaligned $D$ states for thin foils also explains their somewhat lower values of $\eta$ (see Table I).

The decrease of $A_{0}^{\text {col }}$ with the thinnest foils for the $3 p{ }^{1} P$ state most probably results from the slight front-to-back asymmetry in the proton-helium distribution caused by the energy-loss differential (Appendix B). Since the intensity enhancement is due to interactions between $\Sigma$ orbitals, directional alignment of the internuclear axis along the beam direction results in enhancement of the $m_{l}=0$ levels of the separated He atoms, as observed.

The effect of close correlated protons and close correlated secondary electrons on $\mathrm{HeI}$ alignment is seen to be qualitatively different. While protons always reduce the magnitude of $A_{0}^{\text {col }}$, an enhanced electron flux always leads to more positive values of alignment. ${ }^{4-6}$ While the interaction of the fast $\mathrm{He}$ atom with secondary electrons at the surface is quite brief, ${ }^{8}$ the proton-helium interaction is strong for a considerable distance downbeam from the foil. As a result, the information we can obtain about the electron-helium interaction per se from these experiments is limited. Nevertheless, we have shown that close charged particles can alter excited-state alignment. This is certainly a necessary prerequisite for establishing the validity of a secondary electron model as an explanation of the foil-temperature dependence of alignment.

More generally, we note that in typical ionatom collision experiments, one deals with molecular complexes which exist for only $\sim 10^{-16}$ sec. Such collisions are completely diabatic. Moreover, there is a one-to-one correspondence between incident ion energy and the minimum distance of closest approach. In contrast, the collisions, or more accurately, separations with which one deals in a beam-foil experiment of this type occur much more slowly. For extremely thin foils $\left(<1 \mu \mathrm{g} / \mathrm{cm}^{2}\right)$ and high beam velocities, the internuclear separation at foil exit may be limited only by the incident ground-state vibrational distribution. ${ }^{29}$ One can easily choose the emergent separation of the two nuclei by varying either foil thickness or beam energy. Molecular complexes which do not easily lend themselves to traditional colliding beam or beam-gas studies $\left(\mathrm{C}_{2}^{n+}, \mathrm{Li} F^{n+}\right.$, etc. $)$ can in principle be studied with the beam-foil technique. The major disadvantage of this method is the finite center-of-mass (c.m.) energy and emergent separation distributions which result from molecular passage through the foils. Accurate deconvolution of these dis- 
tributions from the data would be necessary before precise information about the transient molecular states could be obtained.

\section{SUMMARY}

We have shown that close dissociated protons affect both the excited-state population and alignment of HeI upon foil exit. Similar effects are seen in the dissociated hydrogenic species as well. Population variations a re caused by diabatic level crossings between different quasimolecular states formed as the proton and helium nucleus exit the foil. The magnitude of alignment when $\mathrm{HeH}^{+}$projectiles are used is always less than or equal to the equivalent atomic projectile value. Incoherent Stark mixing of the HeI excited states in the field of the proton reduces the foil-collision-induced alignment. For $D$ states, where repopulation resulting from level crossing is considerable, the degree of alignment is linked not only to the proton distribution at foil exit directly, but to the extent of excited-state population enhancement as well. The $3 P$ state alignment, on the other hand, being less affected by molecular level crossings, scales well as a simple function of emergent internuclear separation. The beam-foil technique may in the future prove to be a valuable tool for studying ion-atom quasimolecular interactions.

\section{ACKNOWLEDGMENTS}

We thank T. A. Green for supplying us with the numerical results of his calculations of $\mathrm{HeH}^{+}$ excited states, and R. L. Brooks for several helpful discussions. In addition we thank Salvador Tejero for much technical assistance. Professor John Simpson kindly allowed us to use the LASR accelerator at the University of Chicago. This work was supported by the U. S. Department of Energy, Division of Basic Energy Sciences, and the NSF.

\section{APPENDIX A: FOIL THICKENING RATE}

When thin foils a re bombarded by ion beams in vacuum environments which have residual pump oil, they thicken at a rate determined by the chamber pressure, beam energy, and beam current. ${ }^{30}$ In the $\mathrm{HeH}^{+}$experiments, it is important to know the foil thickness so that the average inte rnuclear separation for a given alignment measurement may be determined. The sharp drop of the $2 s^{1} S-3 p^{1} P 5016 \AA$ light intensity with increasing foil thickness below $8 \mu \mathrm{g} / \mathrm{cm}^{2}$ (Fig. 7) provides us with an indirect way to measure foil-thickening rates during bombardment by the beam.

The $5016 \AA$ count rate as a function of time

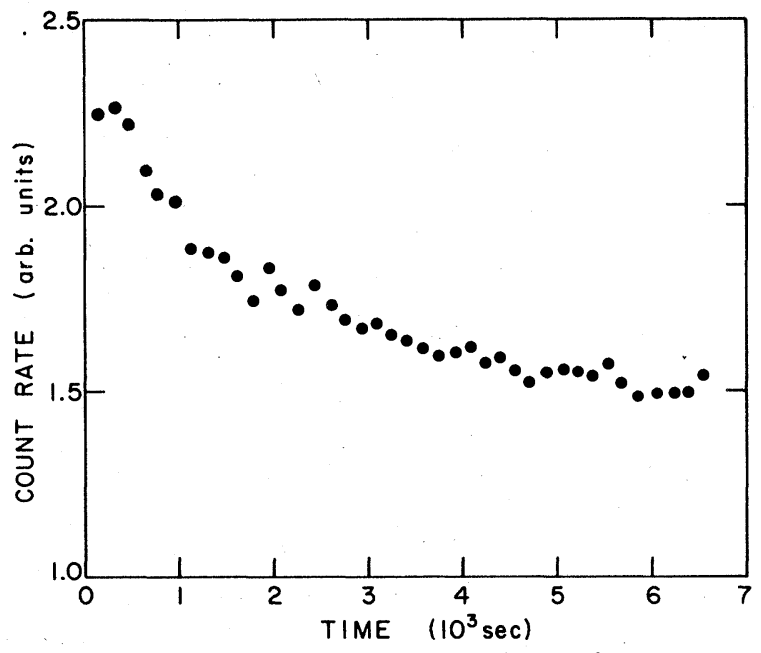

FIG. 11. Relative total light yield of $5016 \AA$ transition vs time of bombardment by $5.6 \mu \mathrm{A} / \mathrm{cm}^{2}, 820-\mathrm{keV}-\mathrm{HeH}^{+}$ beam; initial foil thickness $=1.9 \pm 1 \mu \mathrm{g} / \mathrm{cm}^{2}$, vacuum $=8$ $\times 10^{-7}$ Torr.

for a $5.6-\mu \mathrm{A} / \mathrm{cm}^{2}, 820-\mathrm{keV}-\mathrm{HeH}^{+}$beam on a foil with an initial areal density of $1.9 \pm 1 \mu \mathrm{g} / \mathrm{cm}^{2}$ is shown in Fig. 11. The pressure during this experiment was $8 \times 10^{-7}$ Torr. The intensity drops to two-thirds of its initial value in $6000 \mathrm{sec}$. Referring to Fig. 7, this corresponds to a thickening rate of roughly $20 \mathrm{ng} / \mathrm{cm}^{2} \mathrm{~min}$. The original foil thickness could actually have been between 0.9 and $2.9 \mu \mathrm{g} / \mathrm{cm}^{2}$. This translates to thickening rates between 4 and $60 \mathrm{ng} / \mathrm{cm}^{2}$ min or an increase of 0.1 to $1.5 \mu \mathrm{g} / \mathrm{cm}^{2}$ in a $25 \mathrm{~min}$ run. Assuming a rate somewhere between this, we see that foil thickening does not affect our results significantly.

\section{APPENDIX B: CALCULATION OF THE PROTON- HELIUM INTERNUCLEAR SEPARATION AT FOIL EXIT}

In order to learn something about the length scale for interactions between the dissociated fragments of the $\mathrm{HeH}^{+}$ion as they leave the foil, it is necessary to calculate the ave rage distance between them at foil exit as a function of beam energy and foil thickness. In our case, there are four processes we need to consider to calculate this distance:

(1) Differential energy loss. We assume that the particles lose energy independently. Cluster effects in the stopping power occur when the dynamic screening length is greater than the internuclear separation in the foil. ${ }^{31}$ In our situation, this is true only for the thinnest foils when effects due to differential energy loss are negligible. For a given foil thickness, the proton loses more energy per amu than the helium. For 
thick foils, this results in a front-back asymmetry between the two nuclei.

(2) Multiple scattering from electrons. The particles lose energy in a statistical manner. Hence, even two particles with the same average stopping power will emerge from the foil with some rms front-back separation. In the case of $\mathrm{HeH}^{+}$, this energy straggling smears out the asymmetry due to differential energy loss.

(3) Multiple scattering from nuclei. Angular scattering leads to a roughly Gaussian distribution of internuclear separation along any axis perpendicular to the beam direction.

(4) Coulomb explosion. As a result of electron loss within the first few atomic layers of the foil, the nuclei of the projectile repel each other. When the molecular velocity is small, the two charges are effectively screened and the explosion process is negligible. For higher velocities, the dynamic screening length is larger than the initial internuclear separation and this effect becomes important.

Previous experiments which have investigated molecular effects in the penetration of thin foils by fast ions have measured quantities such as charge fractions and angular distributions of the transmitted atoms or ions. ${ }^{11,32,33}$ Velocities involved in work of this type are typically greater than 5 a.u. In such cases, where the dynamic screening length is much greater than molecular internuclear separations, Coulomb explosion is the dominant dissociative mechanism. As a result, knowledge of dwell time in the foil is sufficient to calculate the internuclear separation at foil exit. For slower velocities, the contributions from multiple scattering become more important. In the velocity regime dictated by optical measurements of neutral helium $(<300$ $\mathrm{keV} / \mathrm{amu}$ ), multiple scattering processes dominate Coulomb effects for all but the thinnest foils at high energy.

For the sake of simplicity in calculation, the various spatial orientations of the incident molecule have not been averaged. The internuclear axis is taken perpendicular to the foil normal. This approximation has little effect on the final calculated emergent separation.

We assume that the molecule loses its electrons instantaneously upon entering the foil and has an initial internuclear separation of $1.4 \mathrm{a} . u .^{34}$ The contributions from the four dissociative processes are calculated independently and added in quadrature. Multiple scattering processes for the two particles are taken to be uncorrelated. This is certainly true for our velocities where the dynamic screening length is small compared to the internuclear separation. We have calculated internuclear separation and molecular centerof-mass energy at foil exit for He exit energies of $125,500,550$, and $650 \mathrm{keV}$ and for foil thicknesses between 2 and $100 \mu \mathrm{g} / \mathrm{cm}^{2}$. $^{2}$

Values for the mean stopping power were obtained from the data of Matteson et al. ${ }^{14}$ at high energy ( $\geqslant 500 \mathrm{keV})$ and from the tables of Northcliffe and Schilling at low energy. ${ }^{15}$ Energy dependence of the stopping power was taken into account by recalculating the energy of the nuclei for every $1 \mu \mathrm{g} / \mathrm{cm}^{2}$ layer of the foil. The calculation starts with a specified energy for the $\mathrm{He}$ at foil exit and works "backwards," adding increments of energy for each microgram of foil thickness. We thus obtain the correct He entrance energy in addition to the time spent in the foil, which is needed for the Coulomb explosion calculations. With this information we calculate the exit energy of the proton as well as the distance it lags behind the $\mathrm{He}$ at foil exit.

The spatial separation of the two nuclei due to energy straggling is calculated assuming that individual energy loss resulted from uniform acceleration or deceleration from the particle's initial velocity. The distribution in energy following traversal of the foil can be considered to be Gaussian with a centroid given by the mean energy loss $\langle d E / d x\rangle$ times the foil thickness. The standard deviation of the Gaussian $\Omega$ is given according to Bohr by

$$
\Omega^{2}=4 \pi Z_{1}^{2} Z_{2} e^{4} N t,
$$

where $Z_{1}$ and $Z_{2}$ are the atomic numbers of the projectile and target, respectively, $N$ is the atomic density of the target, and $t$ is its thickness. ${ }^{35} \mathrm{Chu},{ }^{36}$ using the theory of Bonderup and Hvelplund ${ }^{37}$ has modified Bohr's formula to better describe low-energy ( $<1 \mathrm{MeV} / \mathrm{amu}$ ) straggling. His energy-dependent results for $\Omega^{2}$ have been used in these calculations. It is assumed that $\Omega_{\mathrm{He}}^{2}=4 \Omega_{\mathrm{H}^{\circ}}^{2}{ }^{36}$ As a result, the energy in the center of mass due to energy straggling is given by ${ }^{32}$

$$
\left\langle E_{\text {c.m. }}\right\rangle=\frac{25}{64}\left(\Omega_{\mathrm{He}}^{2} / \bar{E}_{\mathrm{He}}\right)
$$

where $\bar{E}_{\mathrm{He}}$ is the average kinetic energy of the He nucleus in foil. We may also calculate the meansquare internuclear distance along the beam axis due to energy straggling ${ }^{32}$ :

$$
\left\langle d_{\mathrm{ES}}^{2}\right\rangle=\frac{125}{264} \frac{t^{2} \Omega^{2}}{\bar{E}_{\mathrm{He}}^{2}} .
$$

Distribution due to angular scattering is calculated from the theory of Meyer, ${ }^{38}$ which is valid for velocities of the order of 1 a.u. Its predictions for angular half-widths due to multiple scattering in carbon targets have been experimentally verified. ${ }^{16}$ For scattering through a layer of 
thickness $t$, the angular half-width is given by the Meyer theory to be

$$
\theta_{1 / 2}=\left(a \tau+b \tau^{2}\right)^{1 / 2},
$$

where $a$ and $b$ are numerically determined constants depending on the energy, mass, and atomic number of the projectile and the mass and atomic number of the target atoms. These are tabulated by Meyer. The "reduced thickness" $\tau$ is defined as

$$
\tau=\pi \alpha^{2} N t,
$$

where $N$ is the atomic density of the target and $\alpha$ is the Thomas-Fermi screening length. We compute the perpendicular distance from the original particle track to the particle at foil exit assuming half-width scattering through each differential thickness in the foil:

$$
\left\langle d_{\theta}\right\rangle \approx \int_{0}^{t} \theta_{1 / 2}\left(t^{\prime}, E\right) d t^{\prime}
$$

Equation (B6) is not exact because the angular half-width is only approximately equal to the average scattering angle. In a similar manner we calculate the transverse energy of the scattered particle. Doing this for both the $\mathrm{H}$ and $\mathrm{He}$, we determine the average center-of-mass energy and internuclear separation due to angular scattering.

Finally, we estimate the effect of the Coulomb explosion. The Thomas-Fermi screening length $\alpha$ is given by ${ }^{39}$

$$
\alpha=\left(\frac{12 \pi m_{e} e^{2} n}{\hbar^{2}\left(3 \pi^{2} n\right)^{2 / 3}}\right)^{1 / 2},
$$

where $n$ is the number density of electrons in the carbon foil. Assuming each carbon atom contributes four valence electrons to the electron sea and using the Kennedy et $a{ } .^{40}$ value of 1.82 $\mathrm{g} / \mathrm{cm}^{2}$ for the carbon foil density, we obtain a static screening length of 0.82 a.u., considerably less than the ground-state internuclear separation of $\mathrm{HeH}^{+}$(1.4 a.u.). Hence for velocities much less than 1 a.u., the molecule undergoes no Coulomb explosion. In the case of $125-\mathrm{keV}-\mathrm{He}$ exit energy, average velocities in the foil are about 1.1 a.u., corresponding to a dynamic screening length $\beta\left(=v / \omega_{p}\right)$ of 1.2 a.u., still less than the initial $\mathrm{HeH}^{+}$internuclear separation. Hence the effect of Coulomb explosion is considered for only the three high-energy calculations. In these we assume that the explosion takes place with no screening at all until the nuclei are separated by the dynamic screening length. At this point complete shielding is assumed, and the particles drift apart with the velocity they had when their separation equaled $\beta$. The value for $\beta$ is taken to be that corresponding to the entrance velocity of the molecule. In such an approximation, the energy in the center of mass is simply given by

$$
\bar{E}_{\text {c.m. }}(\text { Coulomb explosion })=e^{2}\left(\frac{1}{1.4}-\frac{1}{\beta}\right)
$$

if the nuclei reach a separation equal to or greater than $\beta$ before they exit the foil. For the thinnest foils this does not happen. As a result, we must calculate the internuclear separation $S$ as a function of time $T$ for an unscreened explosion. By integrating the equation of motion we obtain

$T=T_{0}\left\{\epsilon^{1 / 2}(\epsilon-1)^{1 / 2}+\ln \left[\epsilon^{1 / 2}+(\epsilon-1)^{1 / 2}\right]\right\}$,

where

$$
T_{0}=\left(\mu S_{0}^{3} / 2 e^{2}\right)^{1 / 2}, \quad \epsilon=S / S_{0},
$$

$\mu$ is the reduced mass and $S_{0}$ equals the initial internuclear separation. When $S<\beta$, we modify Eq. (B8) accordingly. Determination of the average internuclear separation at foil exit, taking into account the drift time for the thicker foils, is now straightforward.

The results of these calculations for 125 - and 650-keV-He exit energy are given in Tables II and III. The total c.m. energies a re obtained by adding the contributions from the four processes. The internuclear separations are determined by adding distances from the individual calculations in quadrature, and are graphed in Fig. 5. For low energy, angular multiple scattering is the predominant process in determining the spatial distribution of the two nuclei. On the average, the proton lags behind the helium by a considerable amount for thicknesses greater $10 \mu \mathrm{g} / \mathrm{cm}^{2}$, but due to energy straggling, some protons emerge ahead of the $\mathrm{He}$ at all thicknesses. At the higher energies, Coulomb explosion is the dominant process up to $15 \mu \mathrm{g} / \mathrm{cm}^{2}$, although its contribution to the c.m. energy is less important.

The distributions of internuclear separations for thick foils at both energies are quite broad. The rms distance for $650-\mathrm{keV}-\mathrm{He}$ exit energy and $50-\mu \mathrm{g} / \mathrm{cm}^{2}$ foils is $108 \mathrm{a} . \mathrm{u}$., with the proton lagging the He by an average of 40 a.u. However, assuming a Gaussian energy straggling distribution, roughly $30 \%$ of the protons emerge from the foil ahead of the He. The transverse distribution is also broad. This means that in assigning characteristic lengths to specific features of our data (equilibrium thicknesses, for example), we must keep in mind that they are averages of the entire distribution and as such represent upper limits to the length scale for specific processes. In other words, our data are smeared out by the natural spatial distribution of nuclei emerging from the foil. 
*Also at Ryerson Physical Laboratory, University of Chicago, Chicago, Ill. 60637.

$\dagger$ Present address: J. W. Gibbs Laboratory, Yale University, New Haven, Conn. 06520.

${ }^{1}$ H. G. Berry, L. J. Curtis, D. C. Ellis, and R. M. Schectman, Phys. Rev. Lett. 32, 751 (1974).

${ }^{2}$ T. J. Gay, Ph.D. thesis, University of Chicago, 1980 (unpublished).

${ }^{3}$ R. D. Hight, R. M. Schectman; H. G. Berry, G. Gabrielse, and T. Gay, Phys. Rev. A 16, 1805 (1977).

${ }^{4}$ H. G. Berry, G. Gabrielse, T. Gay, and A. E. Livingston, Phys. Scr. 16, 99 (1977).

${ }^{5}$ T. J. Gay and H. G. Berry, Phys. Rev. A 19 , 952 (1979).

${ }^{6}$ T. J. Gay, H. G. Berry, R. DeSerio, H. P. Garnir, R. M. Schectman, N. Schaffel, R. D. Hight, and D. Burns, Phys. Rev. A 23, 1745 (1981).

${ }^{7}$ E. J. Sternglass, Phys. Rev. 108, 1 (1957).

${ }^{8}$ R. A. Baragiola, E. V. Alonso, and A. Oliva Florio, Phys. Rev. D 19, 121 (1979).

${ }^{9}$ T. J. Gay and H. G. Berry; J. Phys. B $\underline{13}$, L199 (1980); see also, Physics With Fast Molecular Ion Beams, edited by D. S. Gemmell, Argonne National Laboratory Internal Report No. ANL/PHY-79-3, 1979 (unpublished).

${ }^{10}$ K. O. Groeneveld, G. Astner, S. Hultberg, S. Mannervik, and P. S. Ramanujam, J. Phys. B 13, L205 (1980).

${ }^{11}$ M. J. Gaillard, J. C. Poizat, A. Ratkowski, J. Remillieux, and A. Auzas, Phys. Rev. A 16, 2323 (1977).

${ }^{12} \mathrm{P}$. Thieberger, in Physics With Fast Molecular Ion Beams, see Ref. 9.

${ }^{13}$ Gerald Gabrielse, Phys. Rev. A 23, 775 (1981).

${ }^{14}$ S. Matteson, E. K. L. Chau, and D. Powers, Phys. Rev. A 14, 169 (1976).

${ }^{15}$ L. C. Northcliffe and R. F. Schilling, Nucl. Data Tables A 7, 233 (1970).

${ }^{16} \mathrm{G}$. Högberg, H. Nordén, and H. G. Berry, Nucl. Instrum. Methods 90, 283 (1970).

${ }^{17} \mathrm{~J}$. B. Marion and F. C. Young, Nuclear Reaction Analysis: Graphs and Tables (North-Holland, Amsterdam, 1968).
${ }^{18}$ T. A. Green, H. H. Michels, J. C. Browne, and M. M. Madsen, J. Chem. Phys. 61, 5186 (1974).

${ }^{19}$ T. A. Green, H. H. Michels, J. C. Browne, and H. H. Madsen, J. Chem. Phys. 61, 5198 (1974).

${ }^{20}$ T. A. Green, H. H. Michels, and J. C. Browne, J. Chem. Phys. 64, 3951 (1976).

${ }^{21}$ T. A. Green, H. H. Michels, and J. C. Browne, J. Chem. Phys. 69, 101 (1978).

${ }^{22}$ W. Lichten, Phys. Rev. 164, 131 (1967).

${ }^{23}$ W. Lichten, Phys. Rev. 131,229 (1963).

${ }^{24}$ B. Dynefors, I. Martinson, and E. Veje, Phys. Scr. 13, 308 (1976).

${ }^{25}$ G. Heine, H. H. Bukow, and H. V. Buttlar, Proceedings of the 5th International Conference on Beam-Foil Spectroscopy, J. Phys. C-1 느, Cl-269 1745 (1979).

${ }^{26} \mathrm{~T}$. A. Green (private communication).

${ }^{27}$ L. D. Landau and E. M. Lifshitz, Quantum Mechanics (Pergamon, Oxford, 1958).

${ }^{28} \mathrm{E}$. Kupfer and H. Winter, Z. Phys. A 285, 3 (1978).

${ }^{29}$ E. P. Kanter, P. J. Cooney, D. S. Gemmell, K.-O. Groeneveld, W. J. Pietch, A. J. Ratkowski, Z. Vager, and B. J. Zabransky, Phys. Rev. A 20, 834 (1979).

${ }^{30}$ P. D. Dumont, A. E. Livingston, Y. Baudinet-Robinet, G. Weber, and L. Quaglia, Phys. Scr. 13, 122 (1976).

${ }^{31} \mathrm{~W}$. Brandt and R. H. Ritchie, Nucl. Instrum. Methods. 132,43 (1976).

${ }^{32}$ W. Escovitz, Ph.D. thesis, University of Chicago, 1979 (unpublished).

${ }^{33} \mathrm{~J}$. Remillieux, in Physics With Fast Molecular Iom Beams, see Ref. 9.

${ }^{34}$ H. H. Michels, J. Chem. Phys. 44, 3834 (1966).

${ }^{35}$ N. Bohr, K. Dan. Vidensk. Selsk., Mat-Fys. Medd. 18, No. 8 (1948)

${ }^{36}$ W. K. Chu, Phys. Rev. A 13, 2057 (1976).

${ }^{37}$ E. Bonderup and P. Hvelplund, Phys. Rev. A $\underline{4}, 562$ (1971).

${ }^{38}$ L. Meyer, Phys. Status. Solidi B 44, 253 (1971).

${ }^{39} \mathrm{C}$. Kittel, Introduction to Solid State Physics (Wiley, New York, 1971).

${ }^{40} \mathrm{E}$. F. Kennedy, D. H. Youngblood, and A. E. Blaugrund, Phys. Rev. 158, 897 (1967). 